\title{
Die Ökonomisierung von Zeit im flexiblen Kapitalismus
}

Kerstin Jürgens

Die Gestaltung der Arbeitszeit steht nach wie vor im Zentrum betrieblicher Arbeitspolitik. Kollektive Vereinbarungen verlieren jedoch zusehends an Prägekraft, während die individuelle zeitliche Regulierung des Arbeitsprozesses an Bedeutung gewinnt. Der Beitrag resümiert die zentralen Merkmale der Arbeitszeitentwicklung in Deutschland und weist auf einen Formwandel der Ökonomisierung von Zeit hin: Es sind die Beschäftigten selbst, die über die effizienz- und leistungsorientierte Zeitverwendung wachen und damit die gewerkschaftliche Arbeits(zeit)politik in neuer Weise herausfordern.

\section{Zeit im Wandel der Arbeitswelt}

Seit den 1990er Jahren wird in arbeitspolitischen Debatten über Tendenzen der „Entgrenzung von Arbeit“ diskutiert. Demnach verlieren im Zuge der Internationalisierung von Produkt-, Dienstleistungs- und Finanzmärkten und einer Vermarktlichung unternehmensinterner Beziehungen nicht nur nationalstaatliche Grenzen an Bedeutung. Auch die Grenzen innerhalb nationaler Ökonomien, zwischen Betrieb und Markt sowie innerhalb betrieblicher Arbeitsorganisation schwinden (Sauer/Döhl 1997; Voß/Pongratz 1998; Moldaschl/Voß 2002; Sauer 2005). Vor allem aktiennotierte Unternehmen orientieren sich an einer Shareholder-Value-Logik und dünnen ihre Personaldecke aus. Rationalisierung und Leistungsverdichtung stehen in Wirtschaftsunternehmen ebenso wie im öffentlichen Dienst auf der Agenda. Damit verbunden sind umfassende Änderungen in der betrieblichen Arbeitsorganisation: Durch Vermarktlichung und Dezentralisierung werden zum einen Konkurrenzprinzipien innerhalb des Unternehmens selbst etabliert. Der Marktdruck wird dadurch unmittelbarer an die Beschäftigten weitergegeben. Zum anderen werden die Regulierung von Arbeitsabläufen und die Verantwortung für das Arbeitsergebnis von Management oder Vorgesetzten auf Arbeitsteams und einzelne Beschäftigte verlagert.

Inwieweit es sich hierbei um eine Erosion von für kapitalistische Arbeitsgesellschaften kennzeichnenden Strukturmerkmalen handelt oder lediglich um Grenzverschiebungen, wird kontrovers eingeschätzt. Ließ sich mit dem Begriff des „Postfordis- mus“ eine „Ablösung“ des bisher vorherrschenden Produktionsmodells assoziieren, betont die These der „Entgrenzung“ ein Nebeneinander von Neuem und Altem, ein Fortwirken fordistischer Prinzipien bei gleichzeitiger Herausbildung dezentraler Arbeitsorganisation, in der sich Beschäftigte Marktlogiken zu eigen machen. Der Begriff der „Entgrenzung“ sensibilisiert insofern für die gegenwärtige Dynamik eines wirtschaftlichen Strukturwandels, der sich nicht auf die wachsende Bedeutung des tertiären Sektors beschränkt, sondern die grundlegenden Prinzipien von Arbeitsorganisation und ihrer gesellschaftlichen Einbettung erfasst.

Betriebliche Zeitregime sind von dieser Entwicklung nicht unberührt geblieben. Die bisherigen Grenzen von Arbeitszeit haben an Prägekraft eingebüßt: Dauer, Lage und Verteilung variieren nicht nur nach Berufsgruppen, sondern dieselbe Person arbeitet mitunter in allen drei Dimensionen hochgradig flexibel. Inzwischen liegt eine ganze Bandbreite von Publikationen vor, in denen die Flexibilisierung von Arbeitszeit in ihren vielschichtigen Facetten ausgeleuchtet wird (z. B. Seifert 2005). Ausgehend von aktuellen Ergebnissen der Arbeitszeitforschung werden im Folgenden zunächst die Dimensionen aufgezeigt, in denen die Flexibilisierung von Arbeitszeit stattfindet. Außerdem werden die Anforderungen identifiziert, die sich aus dieser Flexibilisierung für das Handeln der betroffenen Beschäftigten ergeben. Dadurch rückt der Zusammenhang zwischen der fortschreitenden Flexibilisierung von Arbeitszeit und dem umfassenderen Wandel von Arbeitsorganisation ins Blickfeld. Es soll sichtbar werden, dass die Vermarktlichung von Arbeitsprozessen nicht nur eine nun auch zeitlich flexible Gestaltung des Arbeitseinsatzes induziert bzw. diese vor- aussetzt, sondern darüber hinaus einen Formwandel der Ökonomisierung von Zeit mit sich bringt: Wachten im Industriekapitalismus Vorgesetzte und Stechuhren über die Einhaltung von Arbeitstempo, Pünktlichkeit und Arbeitsdauer, sind es nun die Beschäftigten selbst, die die effektive Nutzung von Zeit kontrollieren. Arbeitspolitische Studien weisen daher vielfach auf eine Verlagerung der Regulierung von Arbeitszeit von ehemals kollektiver Ebene zu einzelnen Beschäftigten hin und problematisieren, dass die Zeitinteressen der Beschäftigten immer weniger durchsetzungsfähig sind. Der gegenwärtige Wandel geht jedoch über einen solchen Akteurswechsel hinaus: Indem neue Arbeitsformen - mehr oder minder explizit - auf eine Internalisierung von Marktlogik zielen, entwickelt sich auch ein neuer Modus zeitlicher Ökonomisierung. Sowohl individuelle Zeitverwendung als auch subjektives Zeiterleben werden dem Prinzip der Effizienzsteigerung unterworfen - und dies beschränkt sich nicht nur auf das Arbeitshandeln in der Erwerbssphäre, sondern strahlt auch auf die Lebensführung insgesamt aus. Die Brisanz des gegenwärtigen Wandels liegt daher nicht lediglich in einer wachsenden Heterogenität von Arbeitszeitrealitäten und einer Individualisierung des Arbeitszeitkonflikts, sondern in den Folgen, die mit einer Internalisierung einseitig ökonomistischer Zeitverwendungslogiken für die Zeitinteressen und das Zeitbewusstsein von Beschäftigten verknüpft sind und ihre Wir-

\footnotetext{
Kerstin Jürgens, $P D$ Dr. phil., Vertretungsprofessorin für Soziologie an der Leibniz Universität Hannover. Arbeitsschwerpunkte: Arbeit, Arbeitsbeziehungen, Familie, Gender, Zeit. e-mail: k.juergens@ish.uni-hannover.de
} 
kung nicht zuletzt auch in den Einstellungen zur gewerkschaftlichen Arbeitszeitpolitik hinterlassen.

\section{Die Ökonomisierung von Zeit im Industrie- kapitalismus}

Vor der Erfindung mechanischer Uhren orientierte sich das Handeln der Menschen überwiegend an den natürlichen Rhythmen, am Wechsel der Jahreszeiten oder der Abfolge der Gezeiten. Es waren zunächst Mönche, die als erste nach einem strengen Diktat der Uhr lebten, eine strikte ZeitRationalität und eine „rationale“ bzw. „methodische" Lebensführung praktizierten (Weber 1988). Den puritanischen Geistlichen galt Zeitvergeudung als erste und schwerste aller Sünden; Geschäftigkeit wurde zur moralischen Pflicht erklärt und als Voraussetzung späteren Seelenheils bewertet (Marx 1961, S. 143; Sombart 1988). Neben religiösen Motiven oder existenziellen Krisenerfahrungen lassen sich maßgebliche Impulse für den Wandel von Zeitstrukturen und Zeitwahrnehmung der ökonomischen Entwicklung zuschreiben. Gilt die Etablierung des Kalenders und damit der Möglichkeiten zeitlicher Koordination als Voraussetzung für die Entwicklung des Handelskapitalismus, so waren Uhren (neben Dampfmaschine und Elektrizität) die Voraussetzung für die Entwicklung des Industriekapitalismus. Arbeiterinnen und Arbeiter wurden in Fabriken auf die Taktungen der Maschinen eingeschworen, doch das Wissen um die Zeit blieb ihnen systematisch entzogen (Thompson 1973). Es war untersagt, Uhren zu tragen; die Kontrolle über die Zeit lag ausschließlich bei den Vorgesetzten. Zeit entwickelte sich dadurch im Industriebetrieb zum Herrschaftsinstrument. Kirchen, Schulen und Fabriken übten auf je spezifische Weise einen Anpassungsdruck an das neue Zeitregime aus. Sie etablierten Zeitordnungen, Rhythmen und Pünktlichkeitsnormen, die den Übergang von der zyklischen Zeit der Agrargesellschaft zur linearen Zeit des Industriekapitalismus einleiteten und die bis heute unsere Gesellschaft prägen. Die Zeitregime dieser zentralen, das Leben der Menschen strukturierenden Institutionen machen die soziale Zeit zum sozialen Zwang.
Das industrielle Zeitregime beschränkte sich jedoch nicht nur auf die Kontrolle von Anwesenheitszeiten, sondern erstreckte sich auch auf den Arbeitsprozess. In Fabriken und Büros wurden monetäre Belohnungen für schnelles Arbeiten eingeführt; die kontinuierliche Beschleunigung entwickelte sich zum vorherrschenden Prinzip von Arbeitsorganisation. Gewinnmaximierung wird bis heute maßgeblich über die Reduzierung der Produktionszeiten erreicht. Im Lohnarbeitsverhältnis liegt daher - Marx' Analyse ist hier noch aktuell - ein grundlegender Konflikt zwischen Arbeit und Kapital begründet, der im Kampf um die Zeit und deren Ökonomisierung seinen Ausdruck findet. Zeitverwendung wird einer einseitigen Effizienz- und Leistungsorientierung unterworfen, die von der Erwerbssphäre auf die Freizeit ausstrahlt. Arbeitskämpfe waren daher stets auch Kämpfe um die Zeit - etwa um die Wochenarbeitszeit oder um den Normalarbeitstag. Arbeitszeiten wurden in der weiteren Entwicklung des Kapitalismus zwar linear verkürzt, doch fand eine weitreichende Intensivierung und Rationalisierung von Arbeit statt, mit der Unternehmen kontinuierliche Produktivitätserhöhungen erzielten.

Der knappe Rekurs auf die Entwicklung von Zeitmessung zeigt: Diese spezifische Form der Ökonomisierung und Objektivierung von Zeit im Industriekapitalismus ist nicht der bloßen Erfindung von Messinstrumenten wie zum Beispiel Uhren geschuldet, sondern entsteht erst durch deren Einsatz als Stechuhren und Kontrollinstrument kleinster Arbeitsschritte durch die Arbeitgeber (Zoll 1982, S. 105). Betriebliche Arbeitszeitorganisation zielte auf eine effizienzorientierte Zeitverwendung, deren Logik durch die Hierarchisierung von Arbeitszeit und Freizeit auch auf andere Lebensbereiche ausstrahlte. Begrenzungen von Arbeitszeit durch Zeitinstitutionen wie Feierabend oder Wochenende entwickelten sich als zeitliche Schutzräume gegenüber der Erwerbssphäre, waren jedoch hinsichtlich ihrer Funktion nicht von dieser getrennt. Freizeit legitimierte sich über die notwendige biologische und soziale Reproduktion von Arbeitskraft. Zeitordnung und Verfahren der Zeitmessung waren eine zentrale Voraussetzung dafür, dass die Ökonomisierung von Zeit derartig vorangetrieben werden konnte. Die Zeitökonomie des Kapitalismus ließ sich jedoch nicht ohne eine entsprechende Praxis der Sub- jekte durchsetzen. Neue Zeiterfahrungen der Subjekte waren und sind insofern zugleich Resultat und Voraussetzung dafür, dass sich der Industriekapitalismus durchsetzen konnte. Die Rationalisierung der Zeitverwendung ist nicht ausschließlich eine Folge der Herausbildung des Kapitalismus, sondern ein wichtiger Wegbereiter.

\section{Kennzeichen der aktuellen Arbeitszeitentwicklung}

Auch heute sind „Zeitfragen“ noch immer „Streitfragen“ (Mönig-Raane 2005). Als arbeitszeitpolitisches Dilemma erweist sich dabei, dass sich Arbeitszeiten zwischen und innerhalb von Branchen und Beschäftigtengruppen so stark ausdifferenzieren, dass sich kollektive Strategien als "sperrig“ und wenig mobilisierungsfähig erweisen. Jede bilanzierende Betrachtung der Arbeitszeitentwicklung in Deutschland läuft daher unweigerlich Gefahr, die Heterogenität des Flexibilisierungsprozesses einzuebnen und damit tendenziell die Reproduktion sozialer Ungleichheit auszublenden. Noch immer unterscheiden sich Quantität und Qualität von Arbeitszeitflexibilisierung für Beschäftigte je nach Ausgestaltung ihrer Arbeits- und Lebensbedingungen und ihres Beschäftigungsverhältnisses. Wie die Arbeitszeit bewertet wird, hängt dabei von den individuellen Zeitpräferenzen der Betroffenen $a b$. Sehen die einen in einer Abschaffung von Zeiterfassung und festgelegten Anwesenheitszeiten einen Gewinn an Zeitsouveränität, erleben andere dies als Auslöser von Belastung. Physische und psychische Beanspruchung, der Grad der Einbindung in Familien- und Sorgearbeit und individuelle Ressourcen, um Anforderungen zu bewältigen, sind Faktoren, die nicht nur diese Bewertung von Arbeitszeiten beeinflussen, sondern Beschäftigten auch je spezifische Optionen des Umgangs mit flexibilisierten Arbeitszeiten eröffnen oder eben verschließen.

Stellt man diese Komplexität von „Zeitfragen" zunächst hintan, lassen sich allgemeinere, die grundlegende Entwicklungsrichtung kennzeichnende Merkmale der gegenwärtigen Arbeitszeitgestaltung identifizieren. Hilfreich ist hier der breite Forschungsstand zum Thema. Zeitbudgeterhebung und Mikrozensus des Statistischen Bundesamtes sowie die Arbeitszeitbericht- 
erstattung für Nordrhein-Westfalen liefern regelmäßig Daten zur Zeitverwendung in Deutschland. Zahlreiche qualitative Studien gehen über die Erhebung von Zeitbudgets hinaus und ergründen sowohl die Ursachen als auch mögliche Folgen veränderter Zeitstrukturen. Diese Erhebungen beinhalten Hinweise auf einen Wandel von Zeitverwendung, der offenkundig weniger auf veränderte Freizeit- oder Konsummuster zurückzuführen ist, als auf Veränderungen in der Arbeitswelt.

\subsection{ACHT ZEITSTRUKTURELLE VERÄNDERUNGEN}

Trotz aller Brüche und Ungleichzeitigkeiten in der Entwicklung lassen sich - so meine These - acht zeitstrukturelle Veränderungen identifizieren, mit denen neue Anforderungen an individuelle Zeitpraxen einhergehen. Berührt dieser Wandel einige Beschäftigtengruppen nur partiell, sind andere gleich mehrfach betroffen und müssen entsprechend umfassende Anpassungsleistungen erbringen, um nicht nur ihren Arbeitseinsatz, sondern auch den Erhalt ihrer Ressource „Arbeitskraft“ zu gewährleisten.

\section{ARBEITSZEIT WIRD EXTENSIVIERT}

Nach einer langen Phase linearer Arbeitszeitverkürzung findet gegenwärtig eine Wiederanhebung der tariflichen Arbeitszeit statt. Diese wird - wie 2006 der Arbeitskampf im öffentlichen Dienst zeigte - nicht als einmalig, sondern seitens der Arbeitgeber als grundlegender „Abschied“ von bisherigen Prämissen proklamiert. Rückblickend wird sich dieser Arbeitskonflikt möglicherweise als tarifpolitischer Wendepunkt erweisen, mit dem die Abkehr von linearer Arbeitszeitverkürzung eingeleitet wurde. Die durchschnittlichen tariflichen Arbeitszeiten lagen zwar schon immer unterhalb der tatsächlichen Arbeitszeiten, doch eine Anhebung der Norm wird die beiden Größen nicht angleichen, sondern die faktischen Anwesenheitszeiten weiter steigern. Im ersten Quartal 2006 betrug die durchschnittliche Arbeitszeit der erwerbstätigen Männer in Deutschland 40,6 Stunden (Eurostat 2006); sie liegt damit im Mittel der EU-25 und fällt höher als z. B. in Frankreich, Großbritannien oder den skandinavischen Ländern aus. ${ }^{1}$ „Kostenlose " Mehrarbeit von Beschäftigten ist „auf dem Vormarsch“ (Anger 2006). Er- werbsverläufe werden ausgedehnt. Obwohl bereits heute viele abhängig Beschäftigte vor dem 65. Lebensjahr in den Ruhestand gehen, wird das Renteneintrittsalter angehoben. ${ }^{2}$

Nimmt der quantitative Umfang von Freizeit ab, so reduziert sich nicht nur der Zeitanteil für notwendige Haus- und Familienarbeit und private Sozialzeit, sondern auch der für alltägliche Regenerationsbedürfnisse zur Verfügung stehende Anteil. Die Brisanz dieser Expansion von Arbeitszeit im Alltag und Lebenslauf entsteht jedoch maßgeblich aus dem Zusammentreffen verlängerter Arbeitszeiten mit den folgenden Merkmalen von Arbeitszeitgestaltung.

\section{ARBEITSZEIT WIRD INTENSIVIERT}

Gegenwärtige Leitlinien betriebswirtschaftlichen Managements sind kontinuierliche Kostensenkungen und Kurzfristigkeit: Eine umfassende Ausschöpfung aktuell vorhandener Arbeitskraft wird gegenüber einer nachhaltigen Personalentwicklung bevorzugt; vor allem im Segment gering qualifizierter Beschäftigung dominiert das Prinzip der Ersetzbarkeit von Arbeitskräften. Daraus folgt eine Intensivierung von Arbeit - und damit auch eine weitere Effizienzorientierung von Zeitverwendung. Zum einen erhöht sich für die Beschäftigten aufgrund von Leistungsvorgaben und Beschleunigung das innerhalb von Zeiteinheiten zu leistende Arbeitspensum. Zum anderen steigt aufgrund von Massenarbeitslosigkeit der Druck, sich der Verdichtung des Arbeitsprozesses auch dann anzupassen, wenn individuelle Belastungssituationen dem entgegenstehen. Langzeitkonten sehen Modelle des Ansparens von Mehrarbeitsstunden über Jahre hinweg vor. Im Jahr 2000 hatten laut Deutschem Industrie- und Handelskammertag (DIHK) bereits $5 \%$ der Großbetriebe solche Konten eingerichtet. Inwieweit diese zukünftig für „Auszeiten“ im Erwerbsverlauf genutzt werden, wird maßgeblich davon abhängen, inwiefern mit solchen Modellen nicht negative Sanktionen einhergehen, wie sie von Teilzeitarbeit bekannt sind (vgl. zur Kontroverse: Bsirske et al. 2005). Auf die nicht nur gesundheitlichen, sondern auch negativen betriebswirtschaftlichen Effekte intensivierter Arbeit weisen Erhebungen zu Pausen hin. Kurze Pausen in kurzen Intervallen erhöhen nicht nur die Leistungsfähigkeit von Beschäftigten, sondern auch die Arbeitszufriedenheit und die Gesundheit (Oppolzer 2006).

\section{ARBEITSZEIT WIRD POLARISIERT}

Während die Mehrarbeitsvolumina Vollzeitbeschäftigter ansteigen, sind weite Teile der Bevölkerung von Arbeitslosigkeit, unfreiwilliger Teilzeitarbeit oder der Abdrängung in geringfügige Beschäftigung betroffen (Seifert 2007). ${ }^{3}$ Der in diesen Gruppen verbreitete Wunsch, die Arbeitszeit auszudehnen, steht in Kontrast zur Diskrepanz zwischen tariflichen, tatsächlichen und gewünschten Arbeitszeiten bei Vollzeitbeschäftigten. Die tatsächlichen Arbeitszeiten liegen sowohl deutlich über den tariflichen als auch über den gewünschten Arbeitszeiten (Bauer/Munz 2005). Physische und psychische Belastungen durch Mehrarbeit müssen insofern nicht nur als faktische Ausdehnung von Arbeitszeit bewerkstelligt, sondern auch in dem Wissen bewältigt werden, dass Umverteilungspotenziale vorhanden sind, aber bislang ungenutzt bleiben. In Verbindung mit wachsender Unsicherheit von Beschäftigungsperspektiven erhöht sich hier der Konkurrenzdruck. Beschäftigte, die noch von den Absicherungen des Normalarbeitsverhältnisses profitieren, sind zunehmend gezwungen, verschlechterte Arbeitsstandards zu akzeptieren. Die wachsende Gruppe von Zeit- und Leiharbeitern, von befristet Beschäftigten und Mini-Jobbern stellt ein Drohpotenzial dar, das von Unternehmen zum Teil gezielt eingesetzt wird, um Leistungsanforderungen zu erhöhen (Dörre 2005). Unter dem

\footnotetext{
Aufgrund der für Deutschland typischen hohen Teilzeitquote von Frauen und deren niedrigen Stundenvolumina sind allgemeine Daten zu den durchschnittlichen Arbeitszeiten aller Beschäftigten nur begrenzt aussagekräftig und bedienen ein "falsches" Bild von Deutschland als „Freizeitweltmeister".

2 Der Verband Deutscher Rentenversicherungsträger gab für 2004 als durchschnittliches Eintrittsalter für Frauen 61,4 Jahre, für Männer 60,8 Jahre an. Brussig/Woitkowski (2006) resümieren eine insgesamt steigende Tendenz und weisen für 2005 rund 62 Jahre für Frauen und Männer aus. Sie sehen jedoch zwei gegensätzliche Trends: Während sich das Renteneintrittsalter erhöht, steigt gleichzeitig der Anteil derjenigen, die bereits mit 60 in Rente gehen.

3 Im Unterschied zu anderen Ländern hat die Erwerbsintegration von Frauen maßgeblich lediglich als "Teilzeit" -Integration stattgefunden. Der Umfang der faktischen Teilzeit fällt dabei weit geringer aus als die gewünschte Arbeitszeit erwerbs tätiger Frauen.
} 
Druck, den Arbeitsplatz zu verlieren, akzeptieren Beschäftigte daher auch eine Lage und Dauer der Arbeitszeit, die den individuellen Interessen und reproduktiven Bedürfnissen zuwider läuft.

\section{ARBEITSZEIT WIRD FLEXIBILISIERT}

Während sich in Deutschland Teilzeitbeschäftigung nach wie vor als ein für Frauen dominantes Arbeitszeitmodell erweist, haben Schicht-, Nacht- und Wochenendarbeit weiter zugenommen. Inzwischen arbeiten 39,6 \% der Beschäftigten in solchen atypischen Arbeitszeiten (vgl. den Beitrag von Groß et al. in diesem Heft). Die gesundheitlichen Belastungen von Arbeitszeiten, die nicht dem Biorhythmus entsprechen, sind breit dokumentiert. Darüber hinaus wird der Personaleinsatz mit dem Instrument des Arbeitszeitkontos nochmals flexibilisiert. Neben der Dauer und Lage ist hier auch die Verteilung von Arbeitszeit an Produktionslogik und Marktschwankungen angepasst. Die Forschung verweist zwar wiederholt auf die Potenziale flexibler Arbeitszeit für eine auch individuell „souveräne“ Gestaltung von Arbeits-, Frei- und Familienzeit. Es muss jedoch geprüft werden, ob die konkreten betrieblichen Modelle faktisch genutzt werden können. Meist sind es nur bestimmte, für das Unternehmen schwer rekrutierbare bzw. „kostenintensive“ Beschäftigtengruppen, für die Maßnahmen zur Reduzierung von Zeitstress initiiert werden. Für alle anderen scheint hingegen eine Rückkehr zu einem „occasionalen Zeitbewusstsein" (Rammstedt 1975) erforderlich, mit dem man sich nun nicht mehr an die natürlichen Widrigkeiten anpasst, sondern eigene, nicht erwerbsbezogene Zeitbedürfnisse und -anforderungen den Zufälligkeiten von Marktgeschehen unterordnet. Die Folgen für die Beschäftigten sind unkalkulierbare Regenerationszeiten sowie ein erhöhter Synchronisations- und Koordinationsaufwand im Privaten.

\section{ARBEITSZEIT WIRD PREKARISIERT}

Das in Betriebsvereinbarungen fixierte Primat der „betrieblichen Belange“ gegenüber individuellen Zeitbedürfnissen mündet für die Beschäftigten häufig in unzuverlässigen und nicht planbaren Arbeitszeiten. „Arbeit auf Abruf" entwickelt sich auch für Beschäftigte im Normalarbeitsverhältnis zum Einsatzprinzip. Für eine wachsende Grup- pe abhängig Beschäftigter beschränken sich die zeitlichen Veränderungen von Erwerbsarbeit jedoch nicht auf einen alltäglich flexiblen Arbeitseinsatz. Sie haben darüber hinaus keine existenzsichernde Arbeitszeitdauer und/oder keine langfristige Beschäftigungsperspektive. Sie sind daher - auch bei vorübergehender Erwerbstätigkeit kontinuierlich arbeitssuchend. Langfristige Planbarkeit oder berufliche Entwicklungschancen sind damit stark eingeschränkt dies betrifft sowohl die Existenzsicherung als auch die soziale Integration oder die Familiengründung. Repräsentative Befragungen bestätigen, dass verlässliches Einkommen und Sicherheit meist schwerer wiegen als konkrete Arbeitsbedingungen (INIFES 2006). In Verbindung mit dem Abbau sozialpolitischer Absicherung und steigenden Mobilitätsanforderungen trägt dies zur Verbreitung von Unsicherheit bei und stellt damit eine Belastungsquelle eigener Art dar. „Zeitliche Prekarität" wird somit ein Ungleichheitsmerkmal auch innerhalb der Gruppe der Vollzeitbeschäftigten.

\section{ARBEITSZEIT WIRD ISOLIERT}

Durch die Verbreitung von Ziel- und Leistungsvereinbarungen ist Zeit kaum noch Gegenstand formaler Arbeitsregulierung. Zeit als Bezugsgröße von Arbeitsorganisation verliert im Vergleich zum Arbeitsergebnis zwar nicht faktisch, aber doch formal an Bedeutung und wird - vor allem im Bereich hoch qualifizierter Beschäftigung - aus Arbeitsvereinbarungen isoliert. 2003 hatten bereits $8 \%$ der Beschäftigten keine Zeiterfassung mehr (Bauer et al. 2004, S. 24). Gleichwohl gewinnt damit Zeit an Bedeutung: Individuelle Zeitkalkulation, Zeitmanagement und kontinuierliche Synchronisationsleistungen innerhalb von und zwischen Abteilungen und Teams kennzeichnen den Arbeitsalltag und sind Voraussetzung, um Leistungsvereinbarungen auszuhandeln. Zeitplanung verliert insofern lediglich formal an Stellenwert, bleibt aber eine der zentralen Dimensionen der Steuerung von Arbeit, die nun jedoch - wie andere Dimensionen auch - von den Beschäftigten selbst zu gewährleisten ist.

\section{ARBEITSZEIT WIRD INDIVIDUALISIERT}

Folge der Arbeitszeitflexibilisierung ist eine Ausdifferenzierung von Arbeitszeit innerhalb von Belegschaften und der Erwerbsbevölkerung. Arbeitszeitkonflikte sind daher nur noch begrenzt kollektive Konflikte, sondern bedürfen - auch vor dem Hintergrund der Erosion kollektiver Interessenvertretung - individueller Regulierung. Da$\mathrm{zu}$ sind nicht nur Verhandlungskompetenzen erforderlich, sondern auch die Fähigkeit, eigene Zeitinteressen zu identifizieren und mögliche Folgewirkungen von Zeitgestaltung abzuschätzen. Angesichts einer wachsenden Selbstverantwortung von Beschäftigten für ihre Weiterbildung ist daher zu erwarten, dass sich in Modellen selbstgesteuerter Arbeitszeit nicht nur bisherige Ungleichheitslagen reproduzieren, sondern sich entlang der vermehrt erforderlichen „soft skills“ neue Polarisierungen ergeben.

Diese sieben Punkte zeigen bereits, dass sich einseitig ökonomische Zeitverwendungslogiken in ganz unterschiedlichen Dimensionen von Arbeitszeitgestaltung durchsetzen. Daraus folgt eine weitreichende Ökonomisierung von Zeit, die sich als indifferent gegenüber reproduktiven Belangen oder disparaten Teilhabechancen erweist. Die zentrale Herausforderung von Arbeitszeitpolitik besteht jedoch - und hiermit greife ich meine Ausgangsthese auf - in einem grundlegenden Wandel im Modus der Ökonomisierung.

\section{ARBEITSZEIT WIRD INTERNALISIERT}

Der historische Rückblick hat gezeigt, dass für die Durchsetzung der betrieblichen Arbeitszeitregime umfassende Disziplinierungsmaßnahmen und Kontrollmechanismen nötig waren. Im Kontext von Dezentralisierung und Vermarktlichung sind derlei Instrumente - vor allem im Bereich (hoch) qualifizierter Beschäftigung - heute weitgehend obsolet (Peters/Sauer 2005). Dies zeigt sich auch in der Dimension „Zeit“. Ebenso wie Markt- und Leistungsorientierung wird auch die optimale Nutzung verfügbarer Arbeitszeit als Handlungslogik internalisiert: qualitativ als dichte und hochgradig effiziente Nutzung verfügbarer Arbeitszeit, quantitativ in Form einer flexibel ausgedehnten Arbeitszeit mit entsprechendem Verzicht auf Sozial- und Erholungszeit. Fallen formale Kontrolle und Zeiterfassung weg, dann ist dies jedoch keineswegs als Abnahme ökonomistischer Zeitverwendungslogiken zu interpretieren, sondern lediglich Indiz für einen Formwandel kapitalistischer Zeitorganisation: Die Ökonomisierung von Zeit wird nicht durch Vorgesetzte, sondern durch die Beschäftigten selbst betrieben. 


\subsection{WEITERE PERSPEKTIVEN}

Diese Synthese zentraler Entwicklungslinien betrieblicher Arbeitszeitregime ließe sich um eine Analyse der Zusammenhänge und Widersprüche zwischen Arbeitszeit, Familienzeit und Sozialzeit ergänzen. Erste Ansatzpunkte einer solchen integrativen Perspektive in politischen Kontexten finden sich im jüngsten Familienbericht (BMFSFJ 2006), in dem Familienleben in seiner Wechselwirkung mit Arbeitszeitgestaltung reflektiert wird. Auch in arbeitspolitischen Debatten taucht vereinzelt die Erkenntnis auf, dass betriebliche Arbeitszeiten in einem engen Geflecht zu anderen Lebensbereichen stehen. In diesen schlagen sich einerseits Folgewirkungen von Flexibilisierung nieder, andererseits ergeben sich aber auch Begrenzungen gegenüber einem umfassenden Zugriff auf den „ganzen Menschen".Verantwortungsübernahme für Sorgearbeit und Prioritätensetzung zugunsten privater Bindungen ziehen zwar nach wie vor negative Sanktionen im Erwerbsleben nach sich, können aber ihrerseits Ressourcen hervorbringen, mit denen Beschäftigte Anforderungen aus der Erwerbssphäre bewältigen oder abwehren können. Sowohl individuell als auch kollektiv sind solche Abgrenzungen hochgradig funktional: Sie gewährleisten nicht nur den Erhalt von Arbeitskraft, sondern auch den Erhalt von Lebenskraft, das heißt, sie festigen die psychische und physische Stabilität der Person und ihre soziale Einbindung. Reproduktion basiert insofern auf eigensinnigen Grenzziehungen der Individuen (vgl. ausführlich: Jürgens 2006).

Anstatt die Arbeitszeitentwicklung nun im Zusammenhang zu Veränderungen in anderen Lebensbereichen zu durchleuchten, möchte ich hier abschließend die Dimension der „Internalisierung “ nochmals aufgreifen. Sie verweist auf einen grundlegenden Formwandel der Ökonomisierung von Zeit. Erklärungsansätze der Arbeitsund Industriesoziologie, die einen neuen Zugriff auf die Nutzung von Arbeitskraft und damit eine neue Logik des entgrenzten Kapitalismus resümieren, lassen sich insofern auch durch Ergebnisse der Arbeitszeitforschung untermauern. Gleichwohl bietet das Thema „Zeit“ den Ansatzpunkt für eine weitergehende Perspektive: Nicht nur der neue Modus der Ökonomisierung von Zeit wird sichtbar, sondern auch die mit ihm verbundenen Entfremdungs- prozesse, die mögliche eigensinnige Grenzziehungen seitens der Beschäftigten konterkarieren.

\section{1 \\ Formwandel der Ökonomi- sierung von Zeit}

Anknüpfend an die historische Entwicklung von Zeitmessung und Zeitvorstellungen lassen sich idealtypisch drei Phasen der Ökonomisierung von Zeit identifizieren.

Die erste Phase lässt sich charakterisieren durch die Abkehr von einer zyklischen, bedürfnisorientierten Zeitverwendung hin zur umfassenden Rationalisierung der Lebensführung. Die Erfindung der Verfahren zur Zeitmessung sowie die Anwendung dieser Instrumente zur Kontrolle des Umfangs und der Produktivität menschlicher Aktivitäten kennzeichnen diese Entwicklung. Entscheidend ist dabei: Zeitlicher Ökonomisierungsdruck wurde in dieser Phase zunächst extern induziert. Er ist Ergebnis von kulturellen Vorgaben, positiven oder negativen Sanktionen oder bedurfte religiöser Begründungen bzw. manifester Kontrollen (Abschnitt 2).

Als zweite Phase lässt sich die Verinnerlichung dieser Zeitverwendungslogik in der fortschreitenden Entwicklung der Industriegesellschaft ausmachen. Indem sich auf breiter Basis eine lineare, quantitative Zeitverwendungslogik durchsetzt, das Zeitbewusstsein prägt und in Vergesellschaftungsprozesse einfließt, werden religiöse oder ökonomische Begründungen oder Maßnahmen der Disziplinierung weitgehend obsolet. Gleichwohl kennzeichnet diese Phase, dass Zeitkonflikte weiterhin sichtbar und kollektiv verankert bleiben. Die Messung von Zeit im Betrieb oder die Regulierung von Zeiten in Tarifverträgen können als sichtbarer Ausdruck von widersprüchlichen Interessen an der Zeit gesehen werden. Gewerkschaften und Betriebsräte setzten Zeitfragen als prominentes Thema auf ihre Agenda und konnten breite Massen für die Verteidigung von Zeitinteressen mobilisieren.

Der Übergang in eine dritte Phase deutet sich nun mit der aktuellen Entwicklung an, die über die bloße Fortsetzung der bekannten Prozesse der Internalisierung zeitlicher Ökonomisierung hinausgeht. Gekennzeichnet ist diese Phase durch ein grundlegend neues Verhältnis von kollekti- ver und individueller Zeit. War es von den Anfängen des Industriekapitalismus bis zum fordistischen Produktionsmodell die kollektive, die soziale Zeit (wie z. B. das betriebliche Arbeitszeitregime), die die Ökonomisierung individueller Zeit vorantrieb, so ist im „flexiblen“ Kapitalismus die individuelle Zeit selbst Instanz des Prozesses. Durch die Abschaffung von Zeiterfassung und die Verweigerung zeitlicher Orientierungsmarken in Leistungsvereinbarungen verlagert sich das Prinzip der Ökonomisierung von Zeit vollständig in die Person hinein. Diese internalisiert nicht nur die Rationalisierungslogik, sondern sie ist darüber hinaus auch selbst das neue "Gegenüber“. Sie muss nicht nur - wie Zeitforschung resümiert - Zeit selbst regulieren, sondern auch den Konflikt zwischen erwerbsbezogenen und lebensweltlich bezogenen Zeitanforderungen austarieren. Kämpfe um die Zeit manifestieren sich entsprechend heute weniger als Auseinandersetzungen kollektiver Akteure oder in Interaktionen, sondern sind in die Person hinein verlagert.

Im entgrenzten Kapitalismus ist es insofern nicht die soziale Zeit, sondern die individuelle Zeit, die sich gegen das Subjekt selbst richtet. Werden Unternehmensinteressen als eigene Interessen der Person definiert, sind es folglich nicht externe Zeitvorgaben, die zu neuen Belastungen führen, sondern die Widersprüchlichkeit von privaten, persönlichen und erwerbsbezogenen eigenen Zeitbedürfnissen. Die Person gerät damit zum einen in Konflikt mit eigenen Potenzialen und Ressourcen, zum anderen werden Erfahrungen des Scheiterns eher als individuelles Defizit, denn als strukturelle Überforderung interpretiert. Diese Folgen sind nicht auf die Handlungsdimension "Zeit" begrenzt. Sie sind grundlegend als Nebenfolgen neuer Formen der Nutzung von Arbeitskraft zu bewerten, als Baustein eines neuen Funktionsprinzips kapitalistischer Arbeitsorganisation (Peters/Sauer 2005). Der Blick auf „Zeit“ geht jedoch über die Offenlegung eines neuen Herrschaftsprinzips hinaus und deckt die Reichweite dieses Wandels auf: Es ist nicht lediglich die individuelle Zeit, also der Modus des Zeithandelns oder die Zeitordnung der Person, die in neuer Weise ,angezapft“ wird, sondern die subjektive Zeit. Gegenstand der Ökonomisierung von Zeit wird der Zeit-Sinn des Menschen - das Zeitgefühl, das Gespür für den rechten Augenblick, für das richtige Tempo, für notwen- 
dige Pausen, das Gespür für die Eigenlogik von Körper- und Sozialzeit. Wird nicht nur die individuelle Zeitverwendung, sondern auch diese subjektive Zeit einer einseitigen Ökonomisierung unterworfen, hat dies weitreichende Folgen: Die Menschen entfremden sich von ihrem Zeit-Sinn. Sie werden damit der Fähigkeit beraubt, eine solche individuelle Zeitordnung $\mathrm{zu}$ entwickeln, mit der sie eine Grenze gegenüber externen Zugriffen aufrechterhalten können. An der Dimension Zeit lässt sich insofern veranschaulichen, dass neue Arbeitsformen nicht nur aufgrund von Angeboten der Selbstverwirklichung und Autonomie von Beschäftigten befürwortet werden, sondern auch aufgrund von Entfremdungserfahrungen, die - explizit oder als Folgeerscheinung - mit der Umstellung auf entgrenzte Arbeit(szeit) verbunden sind.

Die skizzierten Phasen lassen sich als Modell einer historischen Stufenabfolge lesen, der in den einzelnen Phasen eine je homogene Logik der Ökonomisierung von Zeit zugrunde liegt. Dass dies angesichts der Ausdifferenzierung von Arbeitszeitrealitäten kaum einer empirischen Prüfung standhält, haben die Hinweise auf heterogene Arbeitszeitmuster verdeutlicht. Die hier vorgeschlagene Zuspitzung auf ein Phasenmodell soll gleichwohl für einen Wandel in der Ökonomisierungslogik sensibilisieren. Sie bedeutet weder, dass in jeder Phase nur eine Logik der Ökonomisierung von Zeit anzutreffen war bzw. ist, noch ist damit die Entwicklung determiniert. Zum einen lassen sich auch heute Beschäftigtengruppen finden, die noch immer in starren Arbeitszeitregimen mit hoher Fremdkontrolle tätig sind. Gleichzeitig verweisen aber die Ergebnisse der Arbeitszeitforschung auf einen eher umfassenden Wandel von Arbeitszeitregulierung, der sich nicht auf den Bereich hoch qualifizierter Beschäftigung reduziert, sondern auch - wenngleich in abgeschwächter Form den gewerblichen Bereich und untere Qualifikationsstufen erfasst. Zum anderen lässt sich die Frage, inwiefern sich eine grundlegend neue Form zeitlicher Ökonomisierung auf breiter Ebene durchsetzt, nicht ohne Berücksichtigung der Entwicklung kollektiver Interessenvertretung beantworten. Bleibt zumindest der Rahmen von Arbeitszeitgestaltung kollektiv reguliert und gelingt eine Verteidigung bisheriger kollektiver Rhythmen und Zeitinstitutionen, kann dies ebenso eine anhaltende Orientierung individuellen Zeithandelns an kollektiven Zeitnormen bewirken wie auch eine Begrenzung der Ökonomisierung von Zeithandeln insgesamt.

Für Arbeitszeitpolitik und wissenschaftliche Forschung sind die Konsequenzen eines solchen Formwandels der Ökonomisierung von Zeit weitreichend: Finden Konflikte um die Zeit nicht mehr nur zwischen kollektiven Akteuren oder Interaktionspartnern statt, sondern verbleiben sie in der Person selbst, sind diese Konflikte schwerer zu identifizieren und zu regulieren. Weder standardisierte Forschungsverfahren noch traditionelle Interessenvertretungsstrukturen werden diesem Wandel gerecht. Die Sensibilisierung für die strukturellen Ursachen vermeintlich individueller Unfähigkeit bietet ohne Zweifel einen Ausgangspunkt für Prozesse der Solidarisierung auch in solchen Beschäftigtengruppen, die eine vergleichsweise große Distanz zu Gewerkschaften aufweisen. Haben Prozesse der Entfremdung, wie sie oben beschrieben sind, jedoch bereits ihre Wirkung entfaltet, wird die erforderliche Aufklärungsarbeit über die Einrichtung von Kommunikationsforen hinausgehen müssen. Arbeitszeitforschung und -politik sind daher gleichermaßen herausgefordert, ihr Augenmerk nicht nur auf den Wandel von Zeitpraxen und deren Beitrag zur Errichtung „neuer“ Zeitregime zu richten, sondern darüber hinaus die Entwicklung individuellen und kollektiven Zeitbewusstseins zu beobachten. Nicht nur für die Dimension "Zeit" ist eine Aktualisierung von Bewusstseinsforschung erforderlich: Zielt der „neue“ Kapitalismus auf eine generelle Internalisierung seiner Normen durch die Arbeitenden selbst, auf ein doing capitalism, dann bedarf es auch in den kritischen Reflexionen „entgrenzter“ Arbeit eines Perspektivwechsels auf die Subjekte.

\section{Fazit}

Verliert gemessene Zeit in neuen Arbeitsformen an Bedeutung, so lösen sich damit weder grundlegende Interessenkonflikte um die Zeit auf, noch erodiert die Hegemonie einer Logik der Ökonomisierung von Zeit. Zeit bleibt ein Herrschaftsinstrument, doch wandeln sich ganz offenkundig der Modus, in dem dieses eingesetzt wird, sowie der Ort, an dem Interessenkonflikte um die Zeit stattfinden. „Zeit“ erweist sich daher als eine Schlüsselkategorie von Arbeitsforschung. Sie ist nicht lediglich eine Handlungsdimension, an der sich Machtund Herrschaftsverhältnisse aufdecken lassen, sondern sie ist darüber hinaus eine emanzipatorische Kategorie, weil in Zeitpräferenzen stets auch Vorstellungen über eine alternative Lebens- und Arbeitsgestaltung eingewoben sind. Konkrete Zeitutopien sind insofern gerade aufgrund der fortschreitenden Ökonomisierung von Zeit dringlicher denn je. Sie dienen als Projektion einer alternativen gesellschaftlichen Zeitorganisation und setzen eine Analyse solcher Zeitstrukturen und Zeitvorstellungen voraus, die gegenwärtig die Partizipations- und Freiheitsgrade der Individuen einschränken. Hierzu muss jedoch die Arbeitszeitentwicklung auf Prozesse der Internalisierung und Entfremdung hin beleuchtet werden, statt sich auf Möglichkeiten einer Umverteilung von Arbeitszeit oder eine Harmonisierung zeitlicher Taktungen zu beschränken. Kristallisationspunkt von Zeitutopien kann demnach nicht mehr lediglich die Reduzierung der Arbeitszeit sein, sondern die Förderung solcher Zeitpraxen, die sich als Kontrapunkt zur veränderten Ökonomisierungslogik behaupten. Eine emanzipatorische (Arbeits-)Zeitpolitik bedarf daher mehr denn je einer Arbeitsforschung, die den Formwandel zeitlicher Ökonomisierung auf seine Antriebskräfte hin befragt und damit Einsicht in die Entwicklungsdynamik des Kapitalismus und die mit ihm verbundenen sozialen Verwerfungen gibt. 
Anger, S. (2006): Zur Vergütung von Überstunden in Deutschland: Unbezahlte Mehrarbeit auf dem Vormarsch, in: DIW Wochenbericht 15-16, S. $189-196$

Bauer, F./Groß, H./Lehmann, K./Munz, E. (2004): Arbeitszeit 2003. Arbeitszeitgestaltung, Arbeitsorganisation und Tätigkeitsprofile, Köln Bauer, F./Munz, E. (2005): Arbeitszeiten in Deutschland: 40 plus und hochflexibel, in: WSI-Mitteilungen 1, S. 40-48

Bundesministerium für Familie, Frauen und Jugend (BMFSFJ) (2006):

7. Familienbericht. Familie zwischen Flexibilität und Verlässlichkeit, Berlin Brussig, M./Wojtkowski, S. (2006): Durchschnittliches Renteneintrittsalter steigt weiter an, in: Altersübergangs-Report 2, Düsseldorf

Bsirske, F./Mönig-Raane, M./Sterkel, G./Wiedemuth, J. (Hrsg.) (2005):

Perspektive neue Zeitverteilung. Logbuch 2 der ver.di-Arbeitszeitinitiative: Für eine gerechte Verteilung von Arbeit, Zeit und Chancen, Hamburg

Dörre, K. (2005): Prekarität - Eine arbeitspolitische Herausforderung, in: WSI-Mitteilungen 5, S. 250-258

Eurostat (2006): Statistik kurz gefasst 17, S. 1-12

Internationales Institut für empirische Sozialökonomie (INIFES) (2006): Was ist gute Arbeit? Anforderungen aus Sicht von Erwerbstätigen. Forschungsbericht, Stadtbergen

Jürgens, K. (2006): Arbeits- und Lebenskraft. Reproduktion als eigensinnige Grenzziehung, Wiesbaden

Marx, K. (1961): Grundrisse der Kritik der politischen Ökonomie, in: Marx, K./Engels, F.: Werke. Band 42, Berlin (1857/1858)

Moldaschl, M./Voß, G. G. (Hrsg.) (2002): Subjektivierung von Arbeit, München/Mering

Mönig-Raane, M. (Hrsg.) (2005): Zeitfragen sind Streitfragen, Hamburg Oppolzer, A. (2006): Menschengerechte Gestaltung der Arbeit durch Erholzeiten, in: WSI-Mitteilungen 6, S. 321-326
Peters, K./Sauer, D. (2005): Indirekte Steuerung - eine neue Herrschaftsform. Zur revolutionären Qualität des gegenwärtigen Umbruchprozesses, in: Wagner, H. (Hrsg.): ,Rentier' ich mich noch? Neue Steuerungskonzepte im Betrieb, Hamburg, S. 23-58

Rammstedt, O. (1975): Alltagsbewusstsein von Zeit, in: Kölner Zeitschrift für Soziologie und Sozialpsychologie 1, S. 47-63

Sauer, D. (2005): Arbeit im Übergang. Eine Standortbestimmung, Hamburg

Sauer, D./Döhl, V. (1997): Die Auflösung des Unternehmens? - Entwicklungstendenzen der Unternehmensreorganisation in den 90er Jahren, in: ISF München et al. (Hrsg.): Jahrbuch sozialwissenschaftliche Technikberichterstattung 1996, Berlin, S. 19-76

Seifert, H. (Hrsg.) (2005): Flexible Zeiten in der Arbeitswelt, Frankfurt a. M./New York

Seifert, H. (2007): Arbeitszeit - Entwicklungen und Konflikte, in: Aus Politik und Zeitgeschichte 4-5, S. 17-24

Sombart, W. (1988): Der Bourgeois. Zur Geistesgeschichte des modernen Wirtschaftsmenschen, Reinbek (1913)

Thompson, E. P. (1973): Zeit, Arbeitsdisziplin und Industriekapitalismus, in: Braun, R./Fischer, W./Großkreutz, H./Volkmann, H. (Hrsg.): Gesellschaft in der industriellen Revolution, Köln, S. 81-112

Voß, G. G./Pongratz, H. J. (1998): Der Arbeitskraftunternehmer. Eine neue Grundform der Ware Arbeitskraft?, in: Kölner Zeitschrift für Soziologie und Sozialpsychologie 1, S. 131-158

Weber, M. (1988): Gesammelte Aufsätze zur Religionssoziologie I, Tübingen (1920)

Zoll, R. (1982): Zeiterfahrung und Gesellschaftsform, in: Prokla 1, S. 103117 